\title{
Lipidized Prolactin-Releasing Peptide as a New Potential Tool to Treat Obesity and Type 2 Diabetes Mellitus: Preclinical Studies in Rodent Models
}

OPEN ACCESS

Edited by:

Wei Zhao,

City University of Hong Kong, Hong Kong SAR, China

Reviewed by:

Xue-Yan $\mathrm{He}$

Cold Spring Harbor Laboratory,

United States

Maushmi Kumar,

SVKM's Narsee Monjee Institute of

Management Studies, India

*Correspondence:

Lenka Maletínská

maletin@uochb.cas.cz

Specialty section:

This article was submitted to Experimental Pharmacology and Drug

Discovery,

a section of the journal

Frontiers in Pharmacology

Received: 27 September 2021

Accepted: 01 November 2021

Published: 18 November 2021

Citation

Mráziková L, Neprašová $B$, Mengr A

Popelová A, Strnadová V, Holá L,

Železná B, Kuneš $J$ and Maletínská L (2021) Lipidized Prolactin-Releasing Peptide as a New Potential Tool to Treat Obesity and Type 2 Diabetes

Mellitus: Preclinical Studies in

Rodent Models.

Front. Pharmacol. 12:779962.

doi: 10.3389/fphar.2021.779962

\section{Lucia Mráziková ${ }^{1}$, Barbora Neprašová ${ }^{1,2}$, Anna Mengr ${ }^{1}$, Andrea Popelová ${ }^{1}$, Veronika Strnadová ${ }^{1}$, Lucie Holá ${ }^{1}$, Blanka Železná ${ }^{1}$, Jaroslav Kuneš ${ }^{1,2}$ and Lenka Maletínská ${ }^{1 *}$}

${ }^{1}$ Institute of Organic Chemistry and Biochemistry, Czech Academy of Sciences, Prague, Czech, ${ }^{2}$ Institute of Physiology, Czech Academy of Sciences, Praque, Czech

Obesity and type 2 diabetes mellitus (T2DM) are preconditions for the development of metabolic syndrome, which is reaching pandemic levels worldwide, but there are still only a few anti-obesity drugs available. One of the promising tools for the treatment of obesity and related metabolic complications is anorexigenic peptides, such as prolactin-releasing peptide (PrRP). PrRP is a centrally acting neuropeptide involved in food intake and body weight (BW) regulation. In its natural form, it has limitations for peripheral administration; thus, we designed analogs of PrRP lipidized at the N-terminal region that showed high binding affinities, increased stability and central anorexigenic effects after peripheral administration. In this review, we summarize the preclinical results of our chronic studies on the pharmacological role of the two most potent palmitoylated PrRP31 analogs in various mouse and rat models of obesity, glucose intolerance, and insulin resistance. We used mice and rats with diet-induced obesity fed a high-fat diet, which is considered to simulate the most common form of human obesity, or rodent models with leptin deficiency or disrupted leptin signaling in which long-term food intake regulation by leptin is distorted. The rodent models described in this review are models of metabolic syndrome with different severities, such as obesity or morbid obesity, prediabetes or diabetes and hypertension. We found that the effects of palmitoylated PrRP31 on food intake and BW but not on glucose intolerance require intact leptin signaling. Thus, palmitoylated PrRP31 analogs have potential as therapeutics for obesity and related metabolic complications.

Keywords: prolactin-releasing peptide, rodent models, obesity, type 2 diabetes, leptin resistance

\section{INTRODUCTION}

Obesity, along with type 2 diabetes mellitus (T2DM), is reaching pandemic levels worldwide, and both are a prerequisite for the development of metabolic syndrome (MetS), which culminates in an increased risk of metabolic and cardiovascular diseases (Said, Mukherjee, and Whayne 2016; Engin 2017; Tune et al., 2017). Although it is clear that obesity is linked to an unhealthy lifestyle in today's society and that adjusting eating habits and lifestyle can partially address obesity issues, new pharmacological treatments are urgently required. Unfortunately, despite the huge efforts to find 
weight-lowering pharmacotherapies, only a few anti-obesity drugs have recently become available (Rodgers, Tschöp, and Wilding 2012; Kumar 2019; Rose, Bloom, and Tan 2019; Williams, Nawaz, and Evans 2020). One of the promising tools for the treatment of obesity and other related metabolic complications is anorexigenic peptides that are synthetized endogenously in the brain or in the gastrointestinal tract and act centrally to decrease energy intake. As demonstrated in experimental models, these peptides have minimal side effects during long-term anti-obesity treatment (Arch 2015; Patel 2015; Bray et al., 2016).

In their natural form, anorexigenic peptides have several disadvantages for direct use in pharmacotherapy for obesity, mainly due to their chemical instability, short half-life and low brain penetrance through the blood-brain barrier (BBB) after peripheral application. A peptidomimetic approach to modify natural peptides is currently being used for the development of promising drugs (Kumar 2019). The problem of penetration through the BBB can be solved, for example, by coupling of peptides to fatty acids, e.g., palmitic acid, resulting in increased stability and half-life in organisms (Malavolta and Cabral 2011; Salameh and Banks 2014).

Some lipidized peptide-based drugs for treatment of diabetes or obesity have been introduced into the market, such as the insulin analog detemir, which employs myristic acid attached through an amide bound to insulin molecules (Havelund et al., 2004), and liraglutide, a palmitoylated agonist of glucagon-like peptide 1 (GLP-1) (Gault et al., 2011). In addition, liraglutide has been approved for anti-obesity treatment in the United States and Europe (Saxenda). Very recently, a once-weekly injection of the lipidized GLP-1 agonist semaglutide was approved by the FDA for treatment of obesity (Wegovy). Several other peptidomimetics, including multitargeted molecules-dual and triple agonists targeting GLP-1, glucagon and gastric inhibitory polypeptide receptors-are in clinical trials as possible future anti-obesity drugs (Williams, Nawaz, and Evans 2020).

Several neuropeptides of brain origin have been demonstrated to have an anorexigenic effect in animal models, such as prolactin-releasing peptide (PrRP), cocaine- and amphetamine-regulated transcript (CART) peptide, $\alpha$-melanocyte-stimulating hormone ( $\alpha$-MSH) and melaninconcentrating hormone (MCH) (Kunes et al., 2016; Mikulaskova et al., 2016). A lipidized $\alpha$-MSH analog has been shown to be stable and exert a strong anorexigenic effect (Fosgerau et al., 2014); however, further research was terminated because of its adverse effects on the skin (Royalty et al., 2014).

Prolactin-releasing peptide was initially isolated from the hypothalamus as a ligand for the human orphan G-protein coupled receptor (GPR10) (Hinuma et al., 1998) as a possible regulator of prolactin secretion from anterior pituitary cells. However, later findings suggested that prolactin release is likely not a primary function of PrRP (Jarry et al., 2000; Taylor and Samson 2001). Shortly after its discovery, it was established that PrRP has other physiological functions, particularly it has been found to be involved in food intake, body weight (BW) and energy expenditure regulation (Lawrence et al., 2000; Takayanagi et al., 2008; Atanes, Ashik, and Persaud 2021). There are two biologically active isoforms of PrRP, with either 20 (PrRP20) or 31 (PrRP31) amino acids. Both isoforms have a common C-terminal Arg-Phe-amide sequence, which is critical for their biological activity (Roland et al., 1999; Maletínská et al., 2011). The fact that PrRP is involved in food intake and BW regulation is supported by the detection of PrRP and its receptor GPR10 in brain areas involved in food intake regulation, such as several hypothalamic nuclei (e.g., nucleus arcuatus (Arc), paraventricular nucleus (PVN), dorsomedial nucleus (DMN)) and the brainstem (e.g., nucleus tractus solitarius (NTS)). PrRP was also found to have high affinity for the neuropeptide FF2 (NPFF2) receptor, resulting in anorexigenic effects (Engström et al., 2003). It has also been shown in rodents that intracerebroventricular injection of natural PrRP20 and PrRP31 decreased food intake and BW (Lawrence et al., 2000; Ellacott et al., 2003; Maixnerová et al., 2011). Coadministration of PrRP and adipose tissue-born long-term acting regulator of energy balance leptin in rats resulted in additive reductions in nocturnal food intake and BW gain and an increase in energy expenditure (Ellacott et al., 2002).

Furthermore, both GPR10 knockout mice and PrRP-deficient mice developed late-onset obesity and exhibited a significant decrease in energy expenditure compared to wild-type mice (Bjursell et al., 2007) as well as altered insulin sensitivity and lipid homeostasis (Prazienkova et al., 2021). Moreover, PrRPdeficient mice also displayed increased food intake and attenuated responses to food intake, lowering the cholecystokinin (CCK) and leptin signals (Takayanagi et al., 2008). Therefore, PrRP or its receptor(s) might be new targets in obesity treatment.

However, as a centrally released and centrally acting neuropeptide, natural PrRP has several limitations after peripheral administration: low stability in the organism to exert its central effect and inability to reach the target brain receptors. To overcome these disadvantages, we designed analogs of PrRP lipidized at the N-terminal region, which is not essential for biological activity (Maletínská et al., 2015; Kunes et al., 2016). Our earlier studies demonstrated that analogs lipidized by $8-18$ carbon chain fatty acids at the N-terminus of PrRP20 or PrRP31 showed high binding affinities with a $\mathrm{K}_{\mathrm{i}}$ in the nanomolar range for both GPR10 and the NPFF2 receptor, similar to analogs that were palmitoylated through linkers to $\mathrm{Lys}^{11}$ (e.g., palm ${ }^{11}$ PrRP31) (Maletínská et al., 2015; Pražienková et al., 2017; Karnošová et al., 2021).

It was confirmed that lipidization increased the stability of these peptides, as palmitoylated PrRP31 (palm-PrRP31) and myristoylated PrRP20 (myr-PrRP31) were stable for more than $24 \mathrm{~h}$ in rat plasma (Zemenová et al., 2017). In vivo pharmacokinetics studies in mice also showed longer stability for lipidized analogs than for natural, nonlipidized PrRP31 (Maletínská et al., 2015). The long-lasting anorexigenic effect of lipidized analogs of PrRP could be explained by their prolonged stability owing to binding to serum albumin, similar to liraglutide, semaglutide or palmitoylated gastric inhibitory polypeptide (Gault et al., 2011; Lau et al., 2015; Bech et al., 2017). 
Acute in vivo experiments demonstrated that lipidized PrRP analogs have central anorexigenic effects after peripheral administration. Our work further supports several indirect studies confirming that the food intake-lowering effect of these analogs is mainly central. There was a significant and dosedependent decrease in food intake in lean overnight-fasted or freely fed mice after subcutaneous (SC) injection of palm-PrRP31, myr-PrRP20 (Maletínská et al., 2015) or palm ${ }^{11}$-PrRP31 (Pražienková et al., 2017; Pirnik et al., 2021), while analogs lipidized with shorter carbon chains or natural PrRP20 or PrRP31 had no effect on food intake (Maletínská et al., 2015). Moreover, neuronal activity (manifested by increased expression of the immediate early gene c-Fos in brain areas related to food intake regulation) was significantly increased in specific brain nuclei or in areas such as the Arc, PVN, DMN and NTS 90 min after SC application of myr-PrRP20, palm-PrRP31 and palm ${ }^{11}$ PrRP31 but not after natural PrRP31 or octanoyl-PrRP31 administration (Maletínská et al., 2015; Pražienková et al., 2017; Pirník et al., 2018). The central neuronal activation of c-Fos after peripheral application of palmitoylated PrRP is also supported by the selective activation of specific hypothalamic oxytocin and hypocretin neuronal subpopulations both involved in food intake regulation (Pirnik et al., 2015). Furthermore, double c-Fos-GPR10 immunostaining in the brainstem C1/A1 cell group indicated that neurons containing GPR10 receptors are activated after administration of palmitoylated PrRP (Mikulášková et al., 2016).

In the hypothalamus, leptin receptor and PrRP are colocalized and have additive anorexigenic effects. Intracerebroventricular coadministration of PrRP and leptin in rats resulted in additive decrease in food intake and BW loss and an increase in energy expenditure (Ellacott et al., 2002). Furthermore, PrRP-expressing neurons in brain regions involved in food intake regulation (ventromedial nucleus of hypothalamus and ventrolateral medulla and NTS of brainstem) also contain leptin receptors (Ellacott et al., 2002). An anorexigenic effect of PrRP independent of leptin but dependent on the peripheral short-term anorexigenic hormone CCK was suggested in the brainstem. CCK was shown to have no effect on food intake in GPR10knockout mice. This finding suggests that PrRP acting through its receptor may be a key mediator in the central satiating action of CCK (Bechtold and Luckman 2006).

An exogenously influenced CCK system was also shown to be involved in the central anorexigenic effect of peripherally applied palm-PrRP (Pirnik et al., 2021). We can thus hypothesize that peripheral signals (leptin, CCK) and the central neuropeptide PrRP cooperate in the stimulation of food intake-regulating pathways, leading to a decrease in food intake.

In this review, we summarize the preclinical results of our chronic studies on the pharmacological role of the two most potent palmitoylated PrRP31 analogs with the following sequences: $\quad$ palm-PrRP31 (N-palm) SRAHQHSNleETRTPDINPAWYTGRGIRPVGRF-NH $\mathrm{N}_{2}$ ) and palm $^{11}$-PrRP31 (SRTHRHSMEIK(N- $\gamma$-E (N-palm)) TPDINPAWYASRGIRPVGRF- $\mathrm{NH}_{2}$ ).

These analogs were tested in various mouse and rat models of obesity, glucose intolerance/insulin resistance and T2DM resulting from high-fat (HF) diet feeding (diet-induced obesity (DIO) models) or in rodents with nonfunctional leptin signaling due to a spontaneous mutation in the leptin receptor.

Each of these rodent models represents different types and severities of pathological features of MetS, i.e., 1/obesity as shown by increased BW, triacylglycerides, free fatty acids, cholesterol and/or liver steatosis, 2/prediabetes or T2DM as shown by increased glucose and insulin levels and glucose intolerance, 3/ leptin and/or insulin resistance as shown by disrupted peripheral and central leptin or insulin signaling and 4/hypertension as shown by increased blood pressure. All pathologies were compared with that of age-matched control rodents. Chronic peripheral interventions with both palmitoylated PrRP31 analogs in different models allowed us to describe different metabolic changes in these models and to clarify the interactions with other systems involved in food intake regulation, such as the leptin system.

\section{CHRONIC TREATMENT WITH PALMITOYLATED PrRP31 ANALOGS IN MOUSE AND RAT MODELS OF METABOLIC DISEASES}

One of the major risks for the development of cardiovascular and metabolic dysfunction, including obesity, prediabetes and hypertension, is high dietary fat intake. Hypercaloric diets rich in lipids are widely used in experimental studies to induce metabolic disorders commonly found in humans (Dourmashkin et al., 2005; Buettner, Schölmerich, and Bollheimer 2007; Agahi and Murphy 2014). Most rodents tend to become obese and develop pathologies of MetS when fed specific calorie-rich diets (Shafrir, Ziv, and Mosthaf 1999; Bergman et al., 2006; Varga et al., 2010). Frequently used models are mice or rats fed a HF diet.

On the other hand, genetic factors undoubtedly play an important role in obesity development, and it is important to better understand the role of specific factors in food intake regulation using models with genetically disrupted production or signaling of these factors. One of the most important hormones regulating long-term energy balance in organisms is leptin, and the most widely used rodent models of spontaneous genetic obesity and related complications are congenital leptin- or leptin receptor-deficient mice and rats (Varga et al., 2010; Wang, Chandrasekera, and Pippin 2014; Fuchs et al., 2018).

In our studies summarized in this review, various mouse and rat models with different features of MetS were used to investigate the effects of palmitoylated PrRP analogs as potential anti-obesity and antidiabetic compounds and to explore their mechanism of action. Each of these models show a variety of pathologies, and the basic characterization of each model is shown in Table 1.

\section{DIO Models}

DIO rodents are considered models of the most common type of human obesity, which is associated with overconsumption of HF food (Bagnol et al., 2012). To test the effect of chronic treatment 
TABLE 1 | Characterization of rodent models used in studies of interventions with palmitoylated PrRP31 analogs.

\begin{tabular}{|c|c|c|c|c|c|c|c|c|c|c|c|}
\hline Model & \multicolumn{5}{|c|}{ Characterization } & \multicolumn{6}{|c|}{ References } \\
\hline DIO mice C57BL/6J & \multicolumn{5}{|c|}{ Obesity, prediabetes, disturbed central leptin and insulin signaling, liver steatosis } & \multicolumn{6}{|c|}{$\begin{array}{l}\text { (Maletínská et al., 2015; Pražienková et al., 2017; } \\
\text { Holubová et al., 2018) }\end{array}$} \\
\hline DIO rats & \multirow{3}{*}{\multicolumn{5}{|c|}{ Obesity, diabetes, glucose intolerance }} & \multirow{3}{*}{\multicolumn{6}{|c|}{ Holubova et al. (2016); Čermáková et al. (2019) }} \\
\hline Sprague-Dawley and & & & & & & & & & & & \\
\hline Wistar Kyoto & & & & & & & & & & & \\
\hline Ob/ob mice & \multicolumn{5}{|c|}{$\begin{array}{l}\text { Severe early onset obesity, disrupted production of leptin, severe liver steatosis, } \\
\text { glucose intolerance, disturbed central leptin and insulin signaling }\end{array}$} & \multicolumn{6}{|c|}{ Korinkova et al. (2020) } \\
\hline MSG mice & \multicolumn{5}{|c|}{$\begin{array}{l}\text { Obesity, glucose intolerance, hormone disbalance, disrupted hypothalamic leptin and } \\
\text { insulin signaling }\end{array}$} & \multicolumn{6}{|c|}{ Špolcová et al. (2015) } \\
\hline ZDF rats & \multicolumn{5}{|c|}{ Lean, severe T2DM } & \multicolumn{6}{|c|}{ Holubova et al. (2016) } \\
\hline Koletsky rats & \multicolumn{5}{|c|}{$\begin{array}{l}\text { Obesity, prediabetes, hypertension, liver steatosis, disrupted central leptin and insulin } \\
\text { signaling }\end{array}$} & \multicolumn{6}{|c|}{ Mikulaskova et al. (2018) } \\
\hline \multirow[t]{2}{*}{ Model } & \multirow[t]{2}{*}{ Characterization/treatment } & \multirow[t]{2}{*}{ BW change } & \multirow{2}{*}{$\begin{array}{c}\text { Cumulative } \\
\text { food } \\
\text { intake }\end{array}$} & \multirow{2}{*}{$\begin{array}{l}\text { Liver } \\
\text { weight }\end{array}$} & \multirow[t]{2}{*}{ Gluc } & \multirow[t]{2}{*}{ se } & \multirow[t]{2}{*}{ Insulin } & \multirow[t]{2}{*}{ Leptin } & \multirow[t]{2}{*}{ TAG } & \multirow{2}{*}{ CHOL } & \multirow{2}{*}{ FFA } \\
\hline & & & & & & & & & & & \\
\hline DIO C57 & HF vs LF & $\uparrow 63 \%$ & NT & NT & $\uparrow$ & & $\uparrow$ & $\uparrow$ & $\uparrow$ & NT & NS \\
\hline DIO Sprague Dawley & HF vs LF & $\uparrow 22 \%$ & $\downarrow$ & NS & NS & & $\uparrow$ & $\uparrow$ & NS & NT & $\uparrow$ \\
\hline DIO Wistar Kyoto & HF vs LF & $\uparrow 10 \%$ & NT & NS & $\uparrow$ & & NS & NS & NS & NS & NS \\
\hline \multirow[t]{3}{*}{ DIO C57 } & palm-PrRP31 & $\downarrow 13 \%$ & $\downarrow$ & NS & NS & & $\downarrow$ & $\downarrow$ & NT & NT & NT \\
\hline & palm ${ }^{11}$-PrRP31 & $\downarrow 12 \%$ & NS & $\downarrow$ & NS & & $\downarrow$ & $\downarrow$ & $\downarrow$ & $\downarrow$ & $\downarrow$ \\
\hline & palm ${ }^{11}$-PrRP31 & $\downarrow 13,6 \%$ & $\downarrow$ & NS & NS & & NS & $\downarrow$ & NS & $\downarrow$ & NS \\
\hline DIO Sprague Dawley & palm-PrRP31 & $\downarrow 8 \%$ & $\downarrow$ & NS & $\uparrow$ & & NS & NS & NS & NS & NS \\
\hline DIO Wistar Kyoto & palm ${ }^{11}$-PrRP31 & $\downarrow 7,7 \%$ & NT & NS & NS & & NS & NS & NS & NS & NS \\
\hline
\end{tabular}

Statistical analysis was performed by unpaired t-test, significance is shown as increased ( $\uparrow$ ) or decreased ( $\downarrow$ ) vs $L F$ or treatment vs $H F$ saline treated group. Cummulative food intake and body weight (BW) change measured at the end of experiment. Cholesterol (CHOL), free fatty acid (FFA) and triacylglycerides (TAG) measured from the plasma. Non-significant (NS), nottested (NT) (Maletínská et al., 2015; Holubova et al., 2016; Pražienková et al., 2017; Holubová et al., 2018; Mikulaskova et al., 2018; Čermáková et al., 2019; Korinkova et al., 2020).

with palmitoylated PrRP31 analogs on obesity and prediabetes parameters and on temporarily disturbed central leptin and insulin signaling, we used several mouse and rat models in our studies.

C57BL/6 mice fed a HF diet containing $60 \%$ fat based on lard from 8 to 19 weeks of age developed severe obesity and prediabetes (Pelantová et al., 2016). Consumption of the HF diet resulted in significant BW gain in the mice, mediated by an increase in body fat and liver weight and an increased level of leptin, as shown in Table 2. HF diet feeding induced an increase in the mRNA expression of genes involved in lipogenesis in adipose tissue but did not affect the mRNA expression of genes involved in lipolysis. The HF diet also increased the blood glucose level and the insulin and triacylglycerides (TAG) levels in plasma compared to mice on a standard chow diet (LF-low fat diet) (Pelantová et al., 2016).

In the studies of Maletínská (Maletínská et al., 2015) and Pražienkova (Pražienková et al., 2017), C57BL/6 male mice were provided with a HF diet from 8 to 19 weeks of age to induce obesity. Subsequently, mice were treated SC with saline or palmitoylated analogs of PrRP, palm-PrRP31 or palm ${ }^{11}$-PrRP31 twice a day for 2 weeks. Palm-PrRP31 treatment significantly decreased cumulative food intake. Both palm-PrRP31 and palm ${ }^{11}$-PrRP31 significantly decreased BW, which was primarily mediated by a reduction in body fat and liver, accompanied by a decrease in leptin levels (Table 2).
Due to the decrease in mRNA expression of fatty acid synthase (Fasn) in both adipose tissue and the liver along with decreased expression of acetyl-CoA carboxylase (Acaca) and sterol regulatory element-binding protein (Srebp) in the liver, BW reduction most likely resulted from decreased de novo lipogenesis, owing primarily to negative energy balance due to reduced food intake (Maletínská et al., 2015; Pražienková et al., 2017). Moreover, increased uncoupling protein $1(U C P-1)$ mRNA in brown adipose tissue (BAT) after palm ${ }^{11}$-PrRP31 treatment points to a possible increase in energy expenditure. Furthermore, treatment with both palmPrRP31 and palm ${ }^{11}$-PrRP31 significantly lowered insulin levels in the blood of DIO mice, and the levels of free fatty acids (FFAs), cholesterol (CHOL) and TAG were significantly reduced after palm ${ }^{11}$-PrRP31 treatment (Table 2).

The next study of Holubová (Holubová et al., 2018) aimed at palm ${ }^{11}$-PrRP31 posttreatment regarding a possible yo-yo effect after drug termination. C57BL/6 mice were fed for 12 weeks with a HF diet. At the age of 19 weeks, mice were SC injected twice a day with saline for 4 weeks, with palm ${ }^{11}$-PrRP31 for 4 weeks or with palm ${ }^{11}$ PrRP31 for 2 weeks and with saline for the following 2 weeks. DIO mice treated for 4 weeks with palm ${ }^{11}$-PrRP31 and those treated with palm ${ }^{11}$-PrRP31 for 2 weeks and then with saline for 2 weeks reached a similar decrease in BW and body fat and attenuated plasma leptin, which continued for 2 weeks after termination of the 2 weeks-long administration of palm ${ }^{11}$-PrRP31 (Figure 1). 


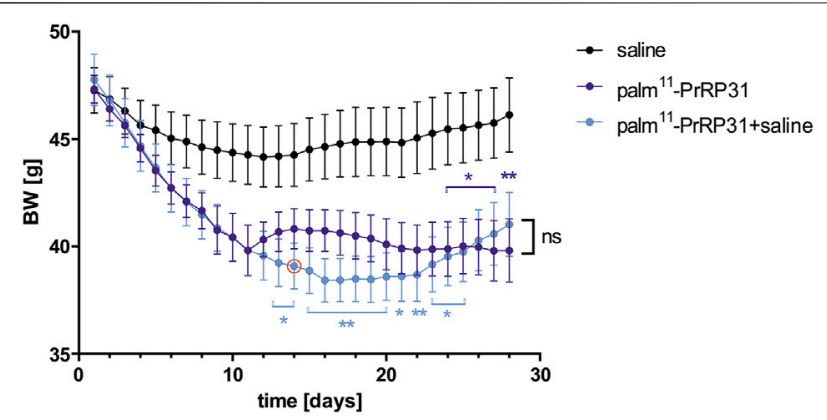

FIGURE 1 | Effect of chronic treatment with palm ${ }^{11}$-PrRP31 on BW in DIO mice. Mice were treated with palm ${ }^{11}$-PrRP31 for 4 weeks (palm ${ }^{11}$-PrRP31 group) or with palm ${ }^{11}$-PrRP31 for 2 weeks and for the following 2 weeks with saline (palm ${ }^{11}$-PrRP31 + saline group), change of the treatment marked by red circle , modified from (Holubová et al., 2018). Data are presented as means \pm S.E.M. Statistical analysis was performed by Two-way ANOVA with Tukey post hoc test, significance is $p<0.05,{ }^{* *}<0.01$ vs saline treated group. Body weight (BW) measured during the treatment, diet-induced obesity (DIO).

mRNA expression of lipolytic enzymes was significantly lowered by the action of palm ${ }^{11}$-PrRP31 in the liver, suggesting complexly attenuated liver lipid metabolism. Furthermore, similar to our previous study, UCP-1 in BAT points to increased energy expenditure. Under both treatment modes, neuronal activity was increased in food intakeregulating neurons, as determined by FosB expression, a marker of long-term neuronal potentiation (Nestler 2001). Blood glucose, insulin, TAG, FFA and CHOL in plasma were not significantly affected by any of the treatments.

Furthermore, in this study, palm ${ }^{11}$-PrRP31 impacted hypothalamic signaling by restoring the leptin receptorinduced phosphatidylinositol-3-kinase (PI3K) pathway and increasing extracellular signal regulated kinase (ERK) $1 / 2$ phosphorylation as a result of increased leptin or PrRP receptor signaling (Balland and Cowley 2015). Moreover, in this study, palm ${ }^{11}$-PrRP31 lowered the phosphorylation of both c-Jun and c-Jun N-terminal kinases (JNKs), generally activated by HF feeding in DIO mice, both in the periphery and the brain (De Souza et al., 2005; Holubová et al., 2018).

Collectively, studies in DIO mouse models revealed a longlasting effect of palmitoylated analogs of PrRP31 on BW lowering, accompanied by increased neuronal signaling in the hypothalamus, even after discontinuation of treatment.

In the following studies, we aimed to examine the effects of intraperitoneal (IP) administration of palmitoylated analogs of PrRP31 in rats fed a HF diet that developed not only severe obesity and prediabetes but also glucose intolerance. Sprague-Dawley rats were provided a HF diet from 8 to 32 weeks and subsequently treated with either saline or palmPrRP31 for 2 weeks (Holubova et al., 2016). Wistar Kyoto rats were fed a HF diet from 8 to 23 weeks of age. At the age of 23 weeks, the mice were IP injected for 3 weeks with either saline or palm ${ }^{11}$-PrRP31 (Čermáková et al., 2019).

The HF diet resulted in significant BW gain, mediated by an increase in body fat and liver weight and an increased level of leptin, as shown in Table 2 (Holubova et al., 2016; Čermáková

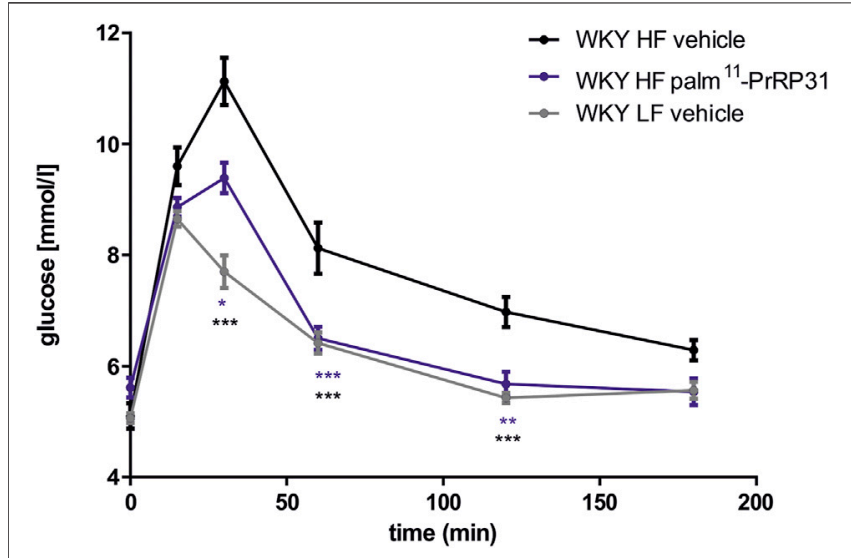

FIGURE 2 | Effect of chronic treatment with palm ${ }^{11}$-PrRP31 on glucose tolerance response in DIO Wistar Kyoto (WKY) rats. Rats were treated with palm ${ }^{11}$-PrRP31 for 3 weeks (WKY HF palm ${ }^{11}$-PrRP31 group). Oral glucose tolerance test (OGTT) was performed after overnight fasting at the end of experiment. Results are shown as a glucose profile modified from (Čermáková et al., 2019). Data are presented as means \pm S.E.M. Statistical analysis was performed by Two-way ANOVA with Tukey post hoc test, significance is $p<0.05,{ }^{* \star}<0.01,{ }^{* \star *}<0.001$ vs WKY HF vehicle treated group. DIO Diet induced obesity, HF high fat, LF low fat.

et al., 2019). Furthermore, the consumption of the HF diet significantly increased intolerance to glucose, determined by an oral glucose tolerance test (OGTT) in both rat strains, with significantly increased fasting blood glucose in Sprague-Dawley rats (Holubova et al., 2016) and increased insulin levels in Wistar Kyoto rats compared to those in the low-fat (LF) diet-fed group.

Similar to mice with DIO, treatment with palm-PrRP31 significantly decreased cumulative food intake, corresponding to a significant decrease in BW in DIO rats after treatment with both palmitoylated analogs (Table 2 ), primarily mediated by a reduction in body fat and liver weight. In these studies, a significant glucose-lowering effect of both PrRP31 analogs was found in DIO rats after the OGTT but not in the saline-treated control group (Figure 2). Treatment with PrRP31 analogs significantly decreased expression of the enzymes that catalyze de novo lipogenesis in both the liver (Holubova et al., 2016; Čermáková et al., 2019) and adipose tissue (Čermáková et al., 2019), while the mRNA expression of lipolytic enzymes was increased after palm ${ }^{11}$-PrRP31 treatment, supporting previous results of complexly affected lipid metabolism. Furthermore, the expression of insulin receptor substrate (Irs) 1 and Irs-2 was increased after palm ${ }^{11}$-PrRP31 treatment. Insulin, TAG, FFA and CHOL in plasma were not significantly affected by any treatment.

In conclusion, in DIO rat models, both palmitoylated analogs of PrRP31 exhibited not only a strong effect on BW lowering but also a great glucose-lowering effect.

\section{Rodent Models With Leptin Deficiency or Disrupted Leptin Signaling}

To test the chronic effect of palmitoylated PrRP31 analogs on obesity and prediabetes or diabetes parameters in relation to the 
TABLE 3 | Summary of metabolic and morphometric parameters in rodent models of leptin deficient or leptin signaling disturbances and impact of treatment with palmPrRP31 or palm ${ }^{11}$-PrRP31.

\begin{tabular}{|c|c|c|c|c|c|c|c|c|c|c|}
\hline Model & Characterization & BW change & $\begin{array}{c}\text { Cumulative } \\
\text { food } \\
\text { intake }\end{array}$ & $\begin{array}{c}\text { Liver } \\
\text { weight }\end{array}$ & Glucose & Insulin & Leptin & TAG & CHOL & FFA \\
\hline$a b / o b$ & ob/ob vs WT & $\uparrow 82 \%$ & NS & $\uparrow$ & NS & $\uparrow$ & NT & NS & $\uparrow$ & NS \\
\hline MSG & MSG vs controls & $\uparrow^{\text {ns }} 7.5 \%$ & NT & NT & NS & $\uparrow$ & $\uparrow$ & NT & NT & NT \\
\hline ZDF & Diabetic ZDF vs non-diabetic ZDF & $\uparrow 10 \%$ & $\uparrow$ & $\uparrow$ & $\uparrow$ & NS & $\uparrow$ & $\uparrow$ & $\uparrow$ & NS \\
\hline SHROB & SHROB vs SHR & $\uparrow 38 \%$ & NS & $\uparrow$ & NS & $\uparrow$ & $\uparrow$ & $\uparrow$ & NS & $\downarrow$ \\
\hline$a b / o b$ & palm ${ }^{11}-\operatorname{PrRP} 31$ & $\downarrow^{\text {ns }} 4 \%$ & NS & NS & NS & NS & NT & NS & $\downarrow$ & NS \\
\hline MSG & palm-PrRP31 & $\downarrow^{\text {ns }} 5.6 \%$ & $\downarrow$ & NT & NS & NS & NS & NT & NT & NT \\
\hline ZDF & palm-PrRP31 & $\downarrow^{\text {ns }} 2 \%$ & $\downarrow$ & NS & NS & NS & NS & NS & $\downarrow$ & NS \\
\hline SHROB & palm ${ }^{11}$-PrRP31 & $\downarrow^{\text {ns }} 1.5 \%$ & $\downarrow$ & NS & NS & $\downarrow$ & NS & NS & NS & $\uparrow$ \\
\hline
\end{tabular}

Statistical analysis was performed by unpaired $\mathrm{t}$-test, significance is shown as increase $(\uparrow)$ or decrease $(\downarrow)$ vs their age-matched controls or treatment vs saline treated group. Cummulative food intake and body weight (BW) change measured at the end of experiment. Cholesterol (CHOL), free fatty acid (FFA) and triacylglycerides (TAG) measured from the plasma. Koletsky rats or spontaneously hypertensive obese rats (SHROB), monosodiumm glutamate (MSG), Non-significant (NS), not-tested (NT), Zucker diabetic fa/fa rats (ZDF), (Maletínská et al., 2015; Holubova et al., 2016; Pražienková et al., 2017; Holubová et al., 2018; Mikulaskova et al., 2018; Čermáková et al., 2019; Korinkova et al., 2020).

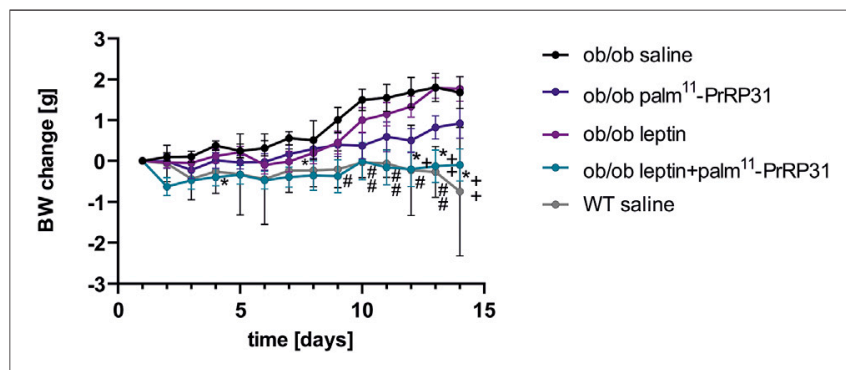

FIGURE 3 | Chronic effect of palm ${ }^{11}$-PrRP31, leptin and their combination on BW change in ob/ob mice modified from (Korinkova et al., 2020) Mice were treated fo 2 weeks. Data are presented as means \pm S.E.M. Statistical analysis was performed by Two-way ANOVA with Tukey post hoc test, significance is ${ }^{\#}<0.05,{ }^{\# \#}<0.01 \mathrm{ob} / \mathrm{ob}$ saline vs wild type (WT) saline, $p<0.05,{ }^{* *}<0.01$ ob/ob leptin + palm ${ }^{11}$-PrRP31 vs ob/ob saline, ${ }^{+}<0.05$, ${ }^{++}<0.01$ ob/ob leptin + palm ${ }^{11}$-PrRP31 vs ob/ob leptin. Body weight (BW) measured during the treatment, wild-type.

important long-term food intake regulator leptin, we used several mouse and rat models with spontaneous leptin deficiency or disrupted leptin signaling.

Leptin-deficient $\mathrm{o} b / o b$ mice were used to explore the potential interaction between leptin and PrRP with regard to their anorexigenic effect and impact on metabolic disturbances (Korinkova et al., 2020). In this study, younger mice (treated from 8 to 10 weeks of age) and older mice (treated from 16 to 24 weeks of age) were used. Younger mice were used because they are in a metabolically active state, and older mice have established morbid obesity.

$\mathrm{Ob} / \mathrm{ob}$ mice of both ages had significantly higher BW, body fat and liver weight than wild-type (WT) mice. As ob/ob mice are known to be hypothermic (Ohtake, Bray, and Azukizawa 1977), their rectal temperature was significantly lower at both 10 and 24 weeks of age. Older $o b / o b$ mice had high hyperinsulinemia and significantly increased cholesterol levels, but TAG and FFA levels did not differ from those of WT mice (Table 3). Our results supported the study of Enser (Enser 1972), who found that $o b / o b$ mice are hyperglycemic only between 5 and 16 weeks of age; however, 24 week-old $o b / o b$ mice were normoglycemic in our study (Korinkova et al., 2020). It was demonstrated that nonfunctional leptin receptor signaling leads to negligible PrRP mRNA expression (Ellacott et al., 2002), suggesting interaction of both systems. In this study, neither palm ${ }^{11}$ PrRP31 nor leptin alone significantly decreased the BW, body fat or liver weight of $o b / o b$ mice, but their combination significantly lowered all these parameters (Figure 3). Moreover, an increase in the rectal temperature in older $o b / o b$ mice was detected after treatment with a combination of leptin and palm ${ }^{11}$-PrRP. Reduced liver weight in younger ob/ob mice treated with a leptin and palm ${ }^{11}$-PrRP31 combination was linked to decreased mRNA expression of lipogenic enzymes in the liver and with regression of fat droplets in liver tissue in all groups of younger peptide-treated $o b / o b$ mice compared to $o b / o b$ salinetreated mice (Korinkova et al., 2020). Treatment with leptin and the combination of palm ${ }^{11}$-PrRP31 + leptin also had a significant decreasing effect on cumulative food intake and total plasma cholesterol levels. In the hypothalamus of older $o b / o b$ mice, two main leptin anorexigenic signaling pathways, namely, Janus kinase (JNK)/signal transducer and activator of transcription-3 (STAT3) activation and AMP-activated protein kinase (AMPK) deactivation, were induced by leptin, palm ${ }^{11}$-PrRP31, and their combination.

Our study (Korinkova et al., 2020) clearly showed that palm ${ }^{11}$ PrRP31 and leptin synergistically lowered BW (Figure 3) and increased body temperature in older $o b / o b$ mice with established morbid obesity. However, the effect of the combination of both drugs on liver weight was only seen in younger $o b / o b$ mice. We can conclude that palm ${ }^{11}$-PrRP31 might be a potential antiobesity drug in the case of a functional leptin system.

MSG mice are a widely used rodent model of obesity and prediabetes. This model is induced by subcutaneous injections of monosodium glutamate (MSG) administered to newborns, resulting in specific lesions in the Arc of the hypothalamus (Takasaki 1978), leading to prediabetes with mild hyperglycemia, hyperinsulinemia and hyperleptinemia (Cameron, Poon, and Smith 1976; Matysková et al., 2008). The obesity of these animals is characterized by increased 
adiposity (Djazayery, Miller, and Stock 1979) because of a lower metabolic rate rather than elevated food intake (Maletínská et al., 2006). We tested whether treatment with palm-PrRP31 influenced the metabolic parameters of the MSG model at 6 months of age when the total adipose tissue weight and plasma level of leptin were significantly higher.

Two weeks of SC treatment with palm-PrRP31 did not significantly change $\mathrm{BW}$ or plasma leptin levels, while the white adipose tissue weight tended to decrease after treatment (Špolcová et al., 2015). While MSG mice were normoglycemic, plasma insulin levels were significantly higher in the MSG mice than in age-matched controls (Table 3). The cumulative food intake was significantly decreased after treatment with palmPrRP31, but the fasting glucose and insulin levels did not differ from those in the saline-treated controls (Table 3). An intraperitoneal glucose tolerance test (IPGTT) showed that only the final glucose level was significantly lower in MSG mice treated with palm-PrRP31 than in MSG mice treated with saline (Špolcová et al., 2015). Moreover, palm-PrRP31 appeared to exert a central anorexigenic effect, resulting in increased phosphorylation of the insulin cascade kinases phosphoinositide-dependent protein kinase 1 (PDK1), protein

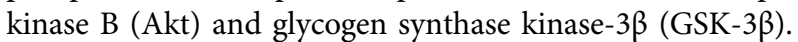

We can conclude that palm-PrRP31 affects metabolic parameters connected with prediabetes in the periphery of MSG mice and insulin signaling in the hippocampus without an effect on BW.

Zucker diabetic rats, which are a model of impaired leptin receptor signaling (Fellmann et al., 2013), are frequently used for studying the potential of antiobesity and antidiabetic peptidic drugs (Andreassen et al., 2014; Skarbaliene et al., 2015). Thus, we used this model to evaluate the chronic antidiabetic potency of palm-PrRP31 and the involvement of the leptin signaling pathway in these effects.

As evident from the definition of this model, ZDF rats were slightly overweight and highly hyperglycemic compared to controls (Holubova et al., 2016). Diabetic ZDF rats had significantly increased cumulative food intake and hyperglycemia and exhibited markedly lowered glucose tolerance during the OGTT in comparison with controls. Hyperlipidemia was also found in diabetic ZDF rats via significantly increased plasma cholesterol and TAG in comparison with controls (Table 3) (Holubova et al., 2016). In this model, 2 weeks of treatment with palm-PrRP31 did not affect BW but had a tendency to improve tolerance to glucose but did not affect fasting glucose. However, the treatment lowered food intake and significantly decreased plasma cholesterol and nonsignificantly decreased plasma free fatty acids, triglycerides, leptin and insulin levels (Table 3).

This study clearly demonstrated that despite the food intakelowering effect, palm-PrRP31 failed to decrease BW or improve glucose tolerance in this model, probably again due to a lack of functional leptin receptors and therefore the impossibility of an interaction of leptin and PrRP systems in the brains of ZDF rats.

The Koletsky rat strain of genetically obese hypertensive rats develops obesity, hyperinsulinemia, hyperlipidemia and spontaneous hypertension, which are the main symptoms of MetS (Koletsky 1973; Xu et al., 2008). These rats showed elevated fasting insulin levels compared to lean spontaneously

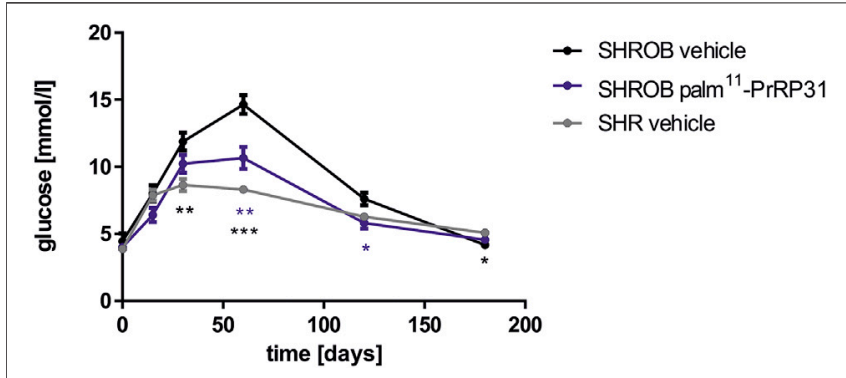

FIGURE 4 | Effect of chronic treatment with palm ${ }^{11}$-PrRP31 on glucose tolerance response in SHROB rats. Rats were treated with palm ${ }^{11}$-PrRP31 for 3 weeks (SHROB palm ${ }^{11}$-PrRP31 group). Oral glucose tolerance test (OGTT) was performed after overnight fasting at the end of experiment. Results are shown as a glucose profile modified from (Mikulaskova et al., 2018). Data are presented as means \pm S.E.M. Statistical analysis was performed by Twoway ANOVA with Tukey post hoc test, significance is $p<0.05,{ }^{* *}<0.01$, ${ }^{* \star *}<0.001$ vs. the vehicle treated control SHR group.

hypertensive rats (SHRs), which were used as a control. OGTTs also demonstrated glucose intolerance; however, the rats were not diabetic, as previously reported (Friedman et al., 1997). Therefore, we tested parameters and insulin signaling in SHROB rats and their SHR controls.

As expected, SHROB rats were obese and had higher leptin, cholesterol and triglyceride levels than SHR controls (Table 3). The level of insulin was significantly higher than that in controls, while both genotypes were normoglycemic (Table 3). SHROB rats showed significantly higher liver weights than SHRs, but kidney and heart weights did not show differences between genotypes in which both were hypertensive (Mikulaskova et al., 2018). The mRNA expression levels of several genes related to lipogenesis in the liver or in adipose tissue were significantly higher in SHROB rats than in SHR controls. Stearoyl-CoA desaturase 1 (Scd-1) contributes to the development of obesity and is suppressed by functional leptin (Biddinger et al., 2006); thus, in this strain with a mutation in the leptin receptor, subcutaneous adipose tissue (SCAT) mRNA expression of Scd-1 was significantly higher in SHROB rats than in SHR controls (Mikulaskova et al., 2018). Treatment with palm ${ }^{11}$-PrRP31 for 3 weeks lowered food intake in both genotypes; however, an effect on BW was seen only in the SHR group with intact leptin signaling. While fasting plasma glucose levels were not affected by treatment in either genotype, based on OGTT results, palm ${ }^{11}$-PrRP31 administration significantly improved tolerance to glucose (Figure 4) in both groups and improved insulin signaling in the hypothalamus (Mikulaskova et al., 2018). The treatment did not have any effect on hypertension in either strain.

The most important result of this study was the marked improvement in glucose tolerance after palm ${ }^{11}$-PrRP treatment in both genotypes, while fasting normoglycemia was not altered. This improvement in glucose tolerance was accompanied by a significant decrease in plasma insulin levels and improved central insulin signaling in SHROB rats. The results also suggested that intact leptin signaling is needed for the BW-lowering effect. 


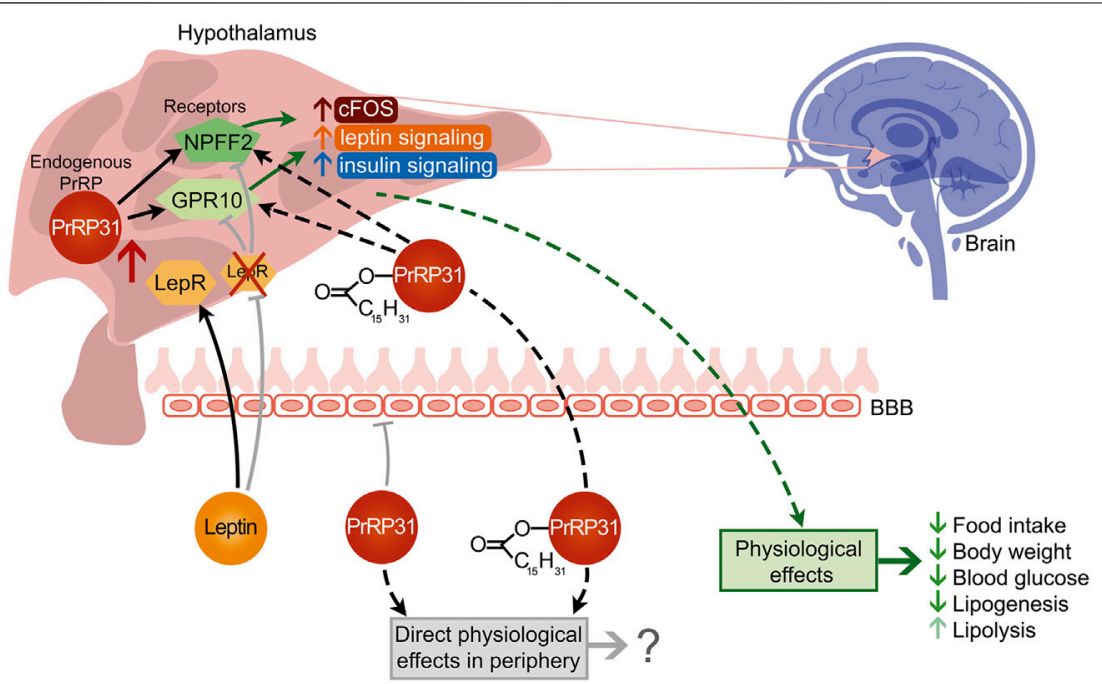

FIGURE 5 | Proposed peripheral and central action of natural PrRP31 and its palmitoylated analog.

\section{CONCLUSION}

This review summarizes our results with a novel potential anorexigenic drug, palmitoylated PrRP, showing its effects on several parameters characterizing obesity or T2DM in different rodent models. Each of these models has specific features and might help us to analyze the particular effects of anorexigenic palm-PrRP analogs and to depict their mechanism of action as potential antiobesity and antidiabetic compounds.

DIO rodent models developed severe obesity, prediabetes or diabetes, resulting in BW gain that was mediated by an increase in body fat and liver weight; in addition, these models showed an increased level of leptin, with disturbed metabolic parameters and increased lipogenesis in adipose tissue. Palm-PrRP31 and palm ${ }^{11}$-PrRP31 seems to reverse the effects of a HF diet. A decrease in food intake resulted in attenuated fat storage and body and liver weight, accompanied by a decrease in leptin levels. Furthermore, palmitoylated analogs of PrRP affected lipid metabolism in adipose tissue and the liver by suppressing lipid synthesis and increasing lipid degradation. Moreover, increased mRNA expression of UCP-1 in BAT points to increased energy expenditure. A very interesting result was also demonstrated in the study after the treatment was discontinued: no yo-yo effect was observed after palm ${ }^{11}$ PrRP31 treatment termination.

The rodent models of leptin deficiency or disturbances in leptin signaling mentioned in this review developed obesity or morbid obesity, but treatment with palm-PrRP31 or palm ${ }^{11}$ PrRP31 did not significantly decrease BW or related metabolic parameters. On the other hand, treatment of $o b / o b$ mice with a combination of leptin and palm ${ }^{11}$-PrRP31 synergistically decreased BW. This synergistic effect was also confirmed by a lower liver weight and body fat and increased body temperature. In two rat strains with nonfunctional leptin signaling, ZDF diabetic rats and Koletsky rats, monotherapy with palm ${ }^{11}$ PrRP31 or palm-PrRP did not have an antiobesity effect, but there were significant glucose-lowering effects. These results suggest that to achieve the full anti-obesity effects of PrRP, intact leptin signaling is needed, but the effect on glucose tolerance could be independent of leptin signaling. The central effect of both palmitoylated PrRP analogs was demonstrated by increased leptin and insulin signaling in the brain.

Overall, based on the results described in this review and in our other studies, the effects of palmitoylated PrRP analogs are summarized in Figure 5. It is evident that natural PrRP is not able to act centrally after peripheral administration and thus affects BW and related metabolic parameters. On the other hand, palmitoylated PrRP stimulates anorexigenic pathways in the hypothalamus. However, our results clearly suggest that the central effects of peripherally applied palm-PrRP on food intake and BW are possible only in the presence of intact leptin signaling. Despite this, palmitoylated PrRP has the potential to be an attractive candidate for obesity therapy.

\section{AUTHOR CONTRIBUTIONS}

LMr, BN, AM did the review search and wrote the manuscript, AP, VS substantially contributed to the writting and editing of manuscript, $\mathrm{LH}$ created the summary figure, BŽ, JK, LMa designed the review, contributed to the writting and edited the manuscript.

\section{FUNDING}

This work was supported by the Grant agency of the Czech Republic No. 21-03691S and by the Czech Academy of Sciences RVO: 61388963 and RVO:67958523. 


\section{REFERENCES}

Agahi, A., and Murphy, K. G. (2014). Models and Strategies in the Development of Antiobesity Drugs. Vet. Pathol. 51, 695-706. doi:10.1177/0300985813492801

Andreassen, K. V., Feigh, M., Hjuler, S. T., Gydesen, S., Henriksen, J. E., BeckNielsen, H., et al. (2014). A Novel Oral Dual Amylin and Calcitonin Receptor Agonist (KBP-042) Exerts Antiobesity and Antidiabetic Effects in Rats. Am. J. Physiol. Endocrinol. Metab. 307, E24-E33. doi:10.1152/ajpendo.00121.2014 Arch, J. R. (2015). Horizons in the Pharmacotherapy of Obesity. Curr. Obes. Rep. 4, 451-459. doi:10.1007/s13679-015-0177-4

Atanes, P., Ashik, T., and Persaud, S. J. (2021). Obesity-induced Changes in Human Islet G Protein-Coupled Receptor Expression: Implications for Metabolic Regulation. Pharmacol. Ther. 228, 107928. doi:10.1016/ j.pharmthera.2021.107928

Bagnol, D., Al-Shamma, H. A., Behan, D., Whelan, K., and Grottick, A. J. (2012). Diet-induced Models of Obesity (DIO) in Rodents. Curr. Protoc. Neurosci. 9, Unit-13. doi:10.1002/0471142301.ns0938s59

Balland, E., and Cowley, M. A. (2015). New Insights in Leptin Resistance Mechanisms in Mice. Front. Neuroendocrinol 39, 59-65. doi:10.1016/j.yfrne.2015.09.004

Bech, E. M., Martos-Maldonado, M. C., Wismann, P., Sørensen, K. K., van Witteloostuijn, S. B., Thygesen, M. B., et al. (2017). Peptide Half-Life Extension: Divalent, Small-Molecule Albumin Interactions Direct the Systemic Properties of Glucagon-like Peptide 1 (GLP-1) Analogues. J. Med. Chem. 60, 7434-7446. doi:10.1021/acs.jmedchem.7b00787

Bechtold, D. A., and Luckman, S. M. (2006). Prolactin-releasing Peptide Mediates Cholecystokinin-Induced Satiety in Mice. Endocrinology 147, 4723-4729. doi:10.1210/en.2006-0753

Bergman, R. N., Kim, S. P., Catalano, K. J., Hsu, I. R., Chiu, J. D., Kabir, M., et al. (2006). Why Visceral Fat Is Bad: Mechanisms of the Metabolic Syndrome. Obesity (Silver Spring) 14 (1), 16s-19s. doi:10.1038/oby.2006.277

Biddinger, S. B., Miyazaki, M., Boucher, J., Ntambi, J. M., and Kahn, C. R. (2006). Leptin Suppresses Stearoyl-CoA Desaturase 1 by Mechanisms Independent of Insulin and Sterol Regulatory Element-Binding Protein-1c. Diabetes 55, 2032-2041. doi:10.2337/db05-0742

Bjursell, M., Lennerås, M., Göransson, M., Elmgren, A., and Bohlooly-Y, M. (2007). GPR10 Deficiency in Mice Results in Altered Energy Expenditure and Obesity. Biochem. Biophys. Res. Commun. 363, 633-638. doi:10.1016/j.bbrc.2007.09.016

Bray, G. A., Frühbeck, G., Ryan, D. H., and Wilding, J. P. (2016). Management of Obesity. Lancet 387, 1947-1956. doi:10.1016/S0140-6736(16)00271-3

Buettner, R., Schölmerich, J., and Bollheimer, L. C. (2007). High-fat Diets: Modeling the Metabolic Disorders of Human Obesity in Rodents. Obesity (Silver Spring) 15, 798-808. doi:10.1038/oby.2007.608

Cameron, D. P., Poon, T. K., and Smith, G. C. (1976). Effects of Monosodium Glutamate Administration in the Neonatal Period on the Diabetic Syndrome in KK Mice. Diabetologia 12, 621-626. doi:10.1007/BF01220641

Čermáková, M., Pelantová, H., Neprašová, B., Šedivá, B., Maletínská, L., Kuneš, J., et al. (2019). 'Metabolomic Study of Obesity and its Treatment with Palmitoylated Prolactin-Releasing Peptide Analog in Spontaneously Hypertensive and Normotensive Rats. J. Proteome Res. 18, 1735-1750.

De Souza, C. T., Araujo, E. P., Bordin, S., Ashimine, R., Zollner, R. L., Boschero, A. C., et al. (2005). Consumption of a Fat-Rich Diet Activates a Proinflammatory Response and Induces Insulin Resistance in the Hypothalamus. Endocrinology 146, 4192-4199. doi:10.1210/en.2004-1520

Djazayery, A., Miller, D. S., and Stock, M. J. (1979). Energy Balances in Obese Mice. Nutr. Metab. 23, 357-367. doi:10.1159/000176281

Dourmashkin, J. T., Chang, G. Q., Gayles, E. C., Hill, J. O., Fried, S. K., Julien, C., et al. (2005). Different Forms of Obesity as a Function of Diet Composition. Int. J. Obes. (Lond) 29, 1368-1378. doi:10.1038/sj.ijo.0803017

Ellacott, K. L., Lawrence, C. B., Pritchard, L. E., and Luckman, S. M. (2003). Repeated Administration of the Anorectic Factor Prolactin-Releasing Peptide Leads to Tolerance to its Effects on Energy Homeostasis. Am. J. Physiol. Regul. Integr. Comp. Physiol. 285, R1005-R1010. doi:10.1152/ajpregu.00237.2003

Ellacott, K. L., Lawrence, C. B., Rothwell, N. J., and Luckman, S. M. (2002). PRLreleasing Peptide Interacts with Leptin to Reduce Food Intake and Body Weight. Endocrinology 143, 368-374. doi:10.1210/endo.143.2.8608

Engin, A. (2017). The Definition and Prevalence of Obesity and Metabolic Syndrome. Adv. Exp. Med. Biol. 960, 1-17. doi:10.1007/978-3-319-48382-5_1
Engström, M., Brandt, A., Wurster, S., Savola, J. M., and Panula, P. (2003). Prolactin Releasing Peptide Has High Affinity and Efficacy at Neuropeptide FF2 Receptors. J. Pharmacol. Exp. Ther. 305, 825-832. doi:10.1124/ jpet.102.047118

Enser, M. (1972). Clearing-factor Lipase in Obese Hyperglycaemic Mice (Ob-ob). Biochem. J. 129, 447-453. doi:10.1042/bj1290447

Fellmann, L., Nascimento, A. R., Tibiriça, E., and Bousquet, P. (2013). Murine Models for Pharmacological Studies of the Metabolic Syndrome. Pharmacol. Ther. 137, 331-340. doi:10.1016/j.pharmthera.2012.11.004

Fosgerau, K., Raun, K., Nilsson, C., Dahl, K., and Wulff, B. S. (2014). Novel a-MSH Analog Causes Weight Loss in Obese Rats and Minipigs and Improves Insulin Sensitivity. J. Endocrinol. 220, 97-107. doi:10.1530/JOE-13-0284

Friedman, J. E., Ishizuka, T., Liu, S., Farrell, C. J., Bedol, D., Koletsky, R. J., et al. (1997). Reduced Insulin Receptor Signaling in the Obese Spontaneously Hypertensive Koletsky Rat. Am. J. Physiol. 273, E1014-E1023. doi:10.1152/ ajpendo.1997.273.5.E1014

Fuchs, T., Loureiro, M. P., Macedo, L. E., Nocca, D., Nedelcu, M., and CostaCasagrande, T. A. (2018). Animal Models in Metabolic Syndrome. Rev. Col Bras Cir 45, e1975. doi:10.1590/0100-6991e-20181975

Gault, V. A., Kerr, B. D., Harriott, P., and Flatt, P. R. (2011). Administration of an Acylated GLP-1 and GIP Preparation Provides Added Beneficial GlucoseLowering and Insulinotropic Actions over Single Incretins in Mice with Type 2 Diabetes and Obesity. Clin. Sci. (Lond) 121, 107-117. doi:10.1042/CS20110006

Havelund, S., Plum, A., Ribel, U., Jonassen, I., Vølund, A., Markussen, J., et al. (2004). The Mechanism of Protraction of Insulin Detemir, a Long-Acting, Acylated Analog of Human Insulin. Pharm. Res. 21, 1498-1504. doi:10.1023/b: pham.0000036926.54824.37

Hinuma, S., Habata, Y., Fujii, R., Kawamata, Y., Hosoya, M., Fukusumi, S., et al. (1998). A Prolactin-Releasing Peptide in the Brain. Nature 393, 272-276. doi:10.1038/30515

Holubová, M., Hrubá, L., Neprašová, B., Majerčíková, Z., Lacinová, Z., Kuneš, J., et al. (2018). Prolactin-releasing Peptide Improved Leptin Hypothalamic Signaling in Obese Mice. J. Mol. Endocrinol. 60, 85-94. doi:10.1530/JME-17-0171

Holubová, M., Zemenová, J., Mikulášková, B., Panajotova, V., Stöhr, J., Haluzík, M., et al. (2016). Palmitoylated PrRP Analog Decreases Body Weight in DIO Rats but Not in ZDF Rats. J. Endocrinol. 229, 85-96. doi:10.1530/JOE-15-0519

Jarry, H., Heuer, H., Schomburg, L., and Bauer, K. (2000). Prolactin-releasing Peptides Do Not Stimulate Prolactin Release In Vivo. Neuroendocrinology 71, 262-267. doi:10.1159/000054544

Karnošová, A., Strnadová, V., Holá, L., Železná, B., Kuneš, J., and Maletínská, L. (2021). Palmitoylation of Prolactin-Releasing Peptide Increased Affinity for and Activation of the GPR10, NPFF-R2 and NPFF-R1 Receptors: In Vitro Study. Int. J. Mol. Sci. 22, 8904. doi:10.3390/ijms22168904

Koletsky, S. (1973). Obese Spontaneously Hypertensive Rats-Aa Model for Study of Atherosclerosis. Exp. Mol. Pathol. 19, 53-60. doi:10.1016/0014-4800(73)90040-3

Korinkova, L., Holubova, M., Neprasova, B., Hruba, L., Prazienkova, V., Bencze, M., et al. (2020). Synergistic Effect of Leptin and Lipidized PrRP on Metabolic Pathways in Ob/ ob Mice. J. Mol. Endocrinol. 64, 77-90. doi:10.1530/jme-19-0188

Kumar, M. S. (2019). Peptides and Peptidomimetics as Potential Antiobesity Agents: Overview of Current Status. Front. Nutr. 6, 11. doi:10.3389/ fnut.2019.00011

Kunes, J., Prazienkova, V., Popelova, A., Mikulaskova, B., Zemenova, J., and Maletinska, L. (2016). Prolactin-releasing Peptide: a New Tool for Obesity Treatment. J. Endocrinol. 230, R51-R58. doi:10.1530/joe-16-0046

Lau, J., Bloch, P., Schäffer, L., Pettersson, I., Spetzler, J., Kofoed, J., et al. (2015). Discovery of the Once-Weekly Glucagon-like Peptide-1 (GLP-1) Analogue Semaglutide. J. Med. Chem. 58, 7370-7380. doi:10.1021/acs.jmedchem.5b00726

Lawrence, C. B., Celsi, F., Brennand, J., and Luckman, S. M. (2000). Alternative Role for Prolactin-Releasing Peptide in the Regulation of Food Intake. Nat. Neurosci. 3, 645-646. doi:10.1038/76597

Maixnerová, J., Špolcová, A., Pýchová, M., Blechová, M., Elbert, T., Rezáčová, M., et al. (2011). Characterization of Prolactin-Releasing Peptide: Binding, Signaling and Hormone Secretion in Rodent Pituitary Cell Lines Endogenously Expressing its Receptor. Peptides 32, 811-817. doi:10.1016/ j.peptides.2010.12.011

Malavolta, L., and Cabral, F. R. (2011). Peptides: Important Tools for the Treatment of central Nervous System Disorders. Neuropeptides 45, 309-316. doi:10.1016/j.npep.2011.03.001 
Maletínská, L., Nagelová, V., Tichá, A., Zemenová, J., Pirník, Z., Holubová, M., et al. (2015). Novel Lipidized Analogs of Prolactin-Releasing Peptide Have Prolonged Half-Lives and Exert Anti-obesity Effects after Peripheral Administration. Int. J. Obes. (Lond) 39, 986-993. doi:10.1038/ijo.2015.28

Maletínská, L., Spolcová, A., Maixnerová, J., Blechová, M., and Zelezná, B. (2011). Biological Properties of Prolactin-Releasing Peptide Analogs with a Modified Aromatic Ring of a C-Terminal Phenylalanine Amide. Peptides 32, 1887-1892. doi:10.1016/j.peptides.2011.08.011

Maletínská, L., Toma, R. S., Pirnik, Z., Kiss, A., Slaninová, J., Haluzík, M., et al. (2006). Effect of Cholecystokinin on Feeding Is Attenuated in Monosodium Glutamate Obese Mice. Regul. Pept. 136, 58-63. doi:10.1016/j.regpep.2006.04.020

Matysková, R., Maletínská, L., Maixnerová, J., Pirník, Z., Kiss, A., and Zelezná, B. (2008). Comparison of the Obesity Phenotypes Related to Monosodium Glutamate Effect on Arcuate Nucleus And/or the High Fat Diet Feeding in C57BL/6 and NMRI Mice. Physiol. Res. 57, 727-734. doi:10.33549/ physiolres. 931274

Mikulaskova, B., Holubova, M., Prazienkova, V., Zemenova, J., Hruba, L., Haluzik, M., et al. (2018). Lipidized Prolactin-Releasing Peptide Improved Glucose Tolerance in Metabolic Syndrome: Koletsky and Spontaneously Hypertensive Rat Study. Nutr. Diabetes 8, 5. doi:10.1038/s41387-017-0015-8

Mikulaskova, B., Maletinska, L., Zicha, J., and Kunes, J. (2016). The Role of Food Intake Regulating Peptides in Cardiovascular Regulation. Mol. Cel Endocrinol 436, 78-92. doi:10.1016/j.mce.2016.07.021

Mikulášková, B., Zemenová, J., Pirník, Z., Pražienková, V., Bednárová, L., Železná, B., et al. (2016). Effect of Palmitoylated Prolactin-Releasing Peptide on Food Intake and Neural Activation after Different Routes of Peripheral Administration in Rats. Peptides 75, 109-117. doi:10.1016/.jpeptides.2015.11.005

Nestler, E. J. (2001). Molecular Neurobiology of Addiction. Am. J. Addict. 10, 201-217. doi:10.1080/105504901750532094

Ohtake, M., Bray, G. A., and Azukizawa, M. (1977). Studies on Hypothermia and Thyroid Function in the Obese (Ob/ob) Mouse. Am. J. Physiol. 233, R110-R115. doi:10.1152/ajpregu.1977.233.3.R110

Patel, D. (2015). Pharmacotherapy for the Management of Obesity. Metabolism 64, 1376-1385. doi:10.1016/j.metabol.2015.08.001

Pelantová, H., Bugáňová, M., Holubová, M., Šedivá, B., Zemenová, J., Sýkora, D., et al. (2016). Urinary Metabolomic Profiling in Mice with Diet-Induced Obesity and Type 2 Diabetes Mellitus after Treatment with Metformin, Vildagliptin and Their Combination. Mol. Cel Endocrinol 431, 88-100. doi:10.1016/ j.mce.2016.05.003

Pirník, Z., Kolesárová, M., Železná, B., and Maletínská, L. (2018). Repeated Peripheral Administration of Lipidized Prolactin-Releasing Peptide Analog Induces C-Fos and FosB Expression in Neurons of Dorsomedial Hypothalamic Nucleus in Male C57 Mice. Neurochem. Int. 116, 77-84. doi:10.1016/ j.neuint.2018.03.013

Pirník, Z., Kořínková, L., Osacká, J., Železná, B., Kuneš, J., and Maletínská, L. (2021). Cholecystokinin System Is Involved in the Anorexigenic Effect of Peripherally Applied Palmitoylated Prolactin-Releasing Peptide in Fasted Mice. Physiol. Res. 70, 579-590. doi:10.33549/physiolres.934694

Pirnik, Z., Železná, B., Kiss, A., and Maletínská, L. (2015). Peripheral Administration of Palmitoylated Prolactin-Releasing Peptide Induces Fos Expression in Hypothalamic Neurons Involved in Energy Homeostasis in NMRI Male Mice. Brain Res. 1625, 151-158. doi:10.1016/j.brainres.2015.08.042

Pražienková, V., Holubová, M., Pelantová, H., Bugáňová, M., Pirník, Z., Mikulášková, B., et al. (2017). Impact of Novel Palmitoylated Prolactin-Releasing Peptide Analogs on Metabolic Changes in Mice with Diet-Induced Obesity. PLoS ONE 12, e0183449-e49. doi:10.1371/journal.pone.0183449

Prazienkova, V., Funda, J., Pirnik, Z., Karnosova, A., Hruba, L., Korinkova, L., et al. (2021). 'GPR10 Gene Deletion in Mice Increases Basal Neuronal Activity, Disturbs Insulin Sensitivity and Alters Lipid Homeostasis. Gene $774,145427$.

Rodgers, R. J., Tschöp, M. H., and Wilding, J. P. (2012). Anti-obesity Drugs: Past, Present and Future. Dis. Model. Mech. 5, 621-626. doi:10.1242/ dmm.009621

Roland, B. L., Sutton, S. W., Wilson, S. J., Luo, L., Pyati, J., Huvar, R., et al. (1999). Anatomical Distribution of Prolactin-Releasing Peptide and its Receptor Suggests Additional Functions in the central Nervous System and Periphery. Endocrinology 140, 5736-5745. doi:10.1210/endo.140.12.7211
Rose, F., Bloom, S., and Tan, T. (2019). Novel Approaches to Anti-obesity Drug Discovery with Gut Hormones over the Past 10 Years. Expert Opin. Drug Discov. 14, 1151-1159. doi:10.1080/17460441.2019.1646243

Royalty, J. E., Konradsen, G., Eskerod, O., Wulff, B. S., and Hansen, B. S. (2014). Investigation of Safety, Tolerability, Pharmacokinetics, and Pharmacodynamics of Single and Multiple Doses of a Long-Acting $\alpha$-MSH Analog in Healthy Overweight and Obese Subjects. J. Clin. Pharmacol. 54, 394-404. doi:10.1002/jcph.211

Said, S., Mukherjee, D., and Whayne, T. F. (2016). Interrelationships with Metabolic Syndrome, Obesity and Cardiovascular Risk. Curr. Vasc. Pharmacol. 14, 415-425. doi:10.2174/1570161114666160722121615

Salameh, T. S., and Banks, W. A. (2014). Delivery of Therapeutic Peptides and Proteins to the CNS. Adv. Pharmacol. 71, 277-299. doi:10.1016/bs.apha.2014.06.004

Shafrir, E., Ziv, E., and Mosthaf, L. (1999). Nutritionally Induced Insulin Resistance and Receptor Defect Leading to Beta-Cell Failure in Animal Models. Ann. N. Y Acad. Sci. 892, 223-246. doi:10.1111/j.1749-6632.1999.tb07798.x

Skarbaliene, J., Secher, T., Jelsing, J., Ansarullah, Neerup, T. S., Billestrup, N., et al. (2015). The Anti-diabetic Effects of GLP-1-Gastrin Dual Agonist ZP3022 in ZDF Rats. Peptides 69, 47-55. doi:10.1016/j.peptides.2015.03.024

Špolcová, A., Mikulášková, B., Holubová, M., Nagelová, V., Pirnik, Z., Zemenová, J., et al. (2015). Anorexigenic Lipopeptides Ameliorate central Insulin Signaling and Attenuate Tau Phosphorylation in Hippocampi of Mice with Monosodium Glutamate-Induced Obesity. J. Alzheimers Dis. 45, 823-835. doi:10.3233/jad-143150

Takasaki, Y. (1978). Studies on Brain Lesion by Administration of Monosodium L-Glutamate to Mice. I. Brain Lesions in Infant Mice Caused by Administration of Monosodium L-Glutamate. Toxicology 9, 293-305. doi:10.1016/0300-483x(78)90013-6

Takayanagi, Y., Matsumoto, H., Nakata, M., Mera, T., Fukusumi, S., Hinuma, S., et al. (2008). Endogenous Prolactin-Releasing Peptide Regulates Food Intake in Rodents. J. Clin. Invest. 118, 4014-4024. doi:10.1172/JCI34682

Taylor, M. M., and Samson, W. K. (2001). The Prolactin Releasing Peptides: RFAmide Peptides. Cell Mol Life Sci 58, 1206-1215. doi:10.1007/PL00000934

Tune, J. D., Goodwill, A. G., Sassoon, D. J., and Mather, K. J. (2017). Cardiovascular Consequences of Metabolic Syndrome. Transl Res. 183, 57-70. doi:10.1016/ j.trsl.2017.01.001

Varga, O., Harangi, M., Olsson, I. A., and Hansen, A. K. (2010). Contribution of Animal Models to the Understanding of the Metabolic Syndrome: a Systematic Overview. Obes. Rev. 11, 792-807. doi:10.1111/j.1467-789X.2009.00667.x

Wang, B., Chandrasekera, P. C., and Pippin, J. J. (2014). Leptin- and Leptin Receptor-Deficient Rodent Models: Relevance for Human Type 2 Diabetes. Curr. Diabetes Rev. 10, 131-145. doi:10.2174/1573399810666140508121012

Williams, D. M., Nawaz, A., and Evans, M. (2020). Drug Therapy in Obesity: A Review of Current and Emerging Treatments. Diabetes Ther. 11, 1199-1216. doi:10.1007/s13300-020-00816-y

Xu, C., Arinze, I. J., Johnson, J., Tuy, T. T., Bone, F., Ernsberger, P., et al. (2008). Metabolic Dysregulation in the SHROB Rat Reflects Abnormal Expression of Transcription Factors and Enzymes that Regulate Carbohydrate Metabolism. J. Nutr. Biochem. 19, 305-312. doi:10.1016/j.jnutbio.2007.05.001

Zemenová, J., Sýkora, D., Maletínská, L., and Kuneš, J. (2017). Lipopeptides as Therapeutics: Applications and In Vivo Quantitative Analysis. Bioanalysis 9, 215-230. doi:10.4155/bio-2016-0206

Conflict of Interest: The authors declare that the research was conducted in the absence of any commercial or financial relationships that could be construed as a potential conflict of interest.

Publisher's Note: All claims expressed in this article are solely those of the authors and do not necessarily represent those of their affiliated organizations, or those of the publisher, the editors and the reviewers. Any product that may be evaluated in this article, or claim that may be made by its manufacturer, is not guaranteed or endorsed by the publisher.

Copyright (c) 2021 Mráziková, Neprašová, Mengr, Popelová, Strnadová, Holá, Železná, Kuneš and Maletínská. This is an open-access article distributed under the terms of the Creative Commons Attribution License (CC BY). The use, distribution or reproduction in other forums is permitted, provided the original author(s) and the copyright owner(s) are credited and that the original publication in this journal is cited, in accordance with accepted academic practice. No use, distribution or reproduction is permitted which does not comply with these terms. 


\section{GLOSSARY}

Acaca acetyl-CoA carboxylase

Akt protein kinase $B$

AMPK AMP-activated protein kinase

Arc nucleus arcuatus

BAT brown adipose tissue

BBB blood brain barrier

BW body weight

CART cocaine- and amphetamine-regulated transcript

CCK cholecystokinin

CHOL cholesterol

DIO diet induced obesity

DMN dorsomedial nucleus

ERK extracellular signal regulated kinase

Fasn fatty-acid synthase

FFA free fatty acid

GLP-1 glucagon-like peptide 1

GPR10 G-protein coupled receptor

GSK-3 $\beta$ glycogen synthase kinase- $3 \beta$

HF high fat

IP intraperitoneal

IPGTT intraperitoneal glucose tolerance test

Irs insulin receptor substrate

JNK c-Jun N-terminal kinase
LF low fat

MCH melanin-concentrating hormone

MetS metabolic syndrome

Myr-PrRP31 myristoylated PrRP

NPFF neuropeptide FF

NPY neuropeptide Y

NTS nucleus tractus solitarii

OGTT oral glucose tolerance test

Palm ${ }^{11}$-PrRP31 palmitoylated PrRP at position 11

Palm-PrRP31 palmitoylated PrRP

PDK1 phosphoinositide-dependent protein kinase 1

PI3K phosphatidylinositol-3-kinase

PrRP prolactin releasing peptide

PVN paraventricular nuclei

SC subcutaneous

SCAT subcutaneous adipose tissue

Scd-1 stearoyl-CoA desaturase-1

Srebp sterol regulatory element-binding protein

STAT3 signal transducer and activator of transcription-3

T2DM type 2 diabetes mellitus

TAG triacylglycerides

UCP-1 uncoupling protein 1

WT wild type

a-MSH a-melanocyte-stimulating hormone 